\title{
A Methodology to Measure Cp / Cv Ratio Using U-shape Acoustic Resonance Tube
}

\author{
Tariq M. Younes ${ }^{1 *}$, Tarek A. Tutunji ${ }^{2}$, Abdel-Hamid Soliman ${ }^{3}$, Ali K. Dalabeeh ${ }^{4}$ \\ ${ }^{1}$ Al Balqa Applied University, Faculty of Engineering Technology, Mechatronics Engineering Department, 19117, Jordan \\ ${ }^{2}$ Philadelphia University, Faculty of Engineering, Mechatronics Engineering Department, 19392, Jordan \\ ${ }^{3}$ Staffordshire University, School of Creative Arts and Engineering (Electrical \& Electronic Engineering), ST18 0AD, UK \\ ${ }^{4}$ Al Balqa Applied University, Faculty of Engineering Technology, Electrical Engineering Department, 19117, Jordan
}

Corresponding Author Email: tariqmog@bau.edu.jo

https://doi.org/10.18280/i2m.180312

Received: 9 March 2019

Accepted: 20 May 2019

\section{Keywords:}

acoustic resonance, $U$-shape resonance tube, specific heat ratio, standing wave

\begin{abstract}
The specific heat ratio of gases can be determined using several measuring instruments. Recently acoustic resonance instruments have shown a great versatility and being used in various branches of industrial applications. This article proposes a simple measurement methodology to measure the specific heat ratio i.e., $\mathrm{Cp} / \mathrm{Cv}$ using free mode of acoustic resonance within a U-shape resonator. The specific heat ratio is calculated by measuring the speed of sound in a tube filled by the sample of the gas under test. For this purpose, the signal of standing wave is acquired using a sound card of Personal Computer. The received signal is processed by LabVIEW software. The experimental results show that the measured value of the specific heat ratio of several types of gases can be calculated using the proposed methodology and the relative error is about $2 \%$.
\end{abstract}

\section{INTRODUCTION}

The development of science and technology over the past few decades have been characterized by an increased interest in thermodynamics and a significant expansion of its applications to various phenomena.

Depending on the conditions under which the heat transfer process was carried out, the body could perform various work. Therefore, the same amount of heat transferred to the body could cause various changes in its internal energy and, therefore, temperature. This ambiguity in the determination of heat capacity is characteristic only for a gaseous substance.

The change in body temperature under heat depends on its chemical composition, mass, and the conditions under which it is heated. To characterize the thermal properties of bodies, the concept of heat capacity was introduced.

Specific heat capacity is one of the most important thermodynamic properties used in engine heat release calculations [1]. This property in may matters defines a massive number of parameters which affects the total efficiency of the engine such as mass flow through the nozzle, gas composition in the engine cylinder. [2]

The importance of the heat capacity was also illustrated during analysis of CANDU postulated accidents, it was also shown that thermal properties of UO21x (i.e., heat capacity and thermal conductivity) cause degradation of the fuel thermal properties [3].

On the other hand, the relations between the work output and the compression ratio, between the thermal efficiency and the compression ratio for an end-reversible Otto cycle are defined by the specific heat ratio of working fluid [4].

Many literatures show that the heat capacity finds wide practical application in many areas, such as: study of the structure of substances and their properties; the study of phase transitions and critical phenomena; calculation of the total amount of impurity in the substance and determining the thermal effects of chemical reactions.

As an example, one can point out the problems of direct or machine-less generation of electrical energy in fuel cells, thermoelectric generators, thermionic converters and magneto-gas-dynamic generators.

This work proposes a novel technique to measure the heat capacity ratio of gases using free mode of acoustic resonance detector. The main features of the developed computerized detector are its simplicity and availability to work without any additional interface to a personal computer.

\section{LITERATURE REVIEW}

The heat capacity of a gas depends on its heating conditions. In particular, the gas can be heated at a constant volume $\mathrm{V}$ or at a constant pressure P. Accordingly, we obtain the heat capacity at a constant volume $\mathrm{Cv}$ and the heat capacity at constant pressure $\mathrm{Cp}$. The ratio $\mathrm{Cp} / \mathrm{Cv}=\gamma$ is a parameter in adiabatic processes ( $\gamma$ is called the Poisson coefficient) [5].

The heat capacity of gases can be measured using various devices and methods. The most common apparatus is Clement and Désormes Apparatus [6]. The principle of determining the adiabatic constant is as follows: Air is pumped into the cylinder to a pressure slightly higher than atmospheric (pressure difference is controlled by a pressure gauge). Then, the air is held in the balloon for a certain period of time. The temperature of the air in the cylinder due to heat transfer becomes equal to the ambient temperature. Because the mass of the gas changes during the experiment, it is convenient to carry out calculations for one mole of gas [7]. Although this method is widely used to determine specific heat capacity, but it has a serious disadvantage; if the closure of the stopcock is large enough to allow rapid expansion, vibrating the air inside 
the bottle, it is not possible to recognize the closure of the stopcock during closure, because this must be done after the expansion stage.

In work [8], the authors utilize Rucchart's method to determine the value $\mathrm{C}_{\mathrm{P}} / \mathrm{C}_{\mathrm{V}}$ of air using the presence of water and its vapors in a vessel. They demonstrated the roles of water evaporation and condensation in the measurements. Their work also showed how to take into account appropriate amendments $[9,10]$. The main disadvantages of the method are the complexity of apparatus and the effect of the pressure inside the tube [11].

In Ref. [12], the authors utilize the speed of sound to determine the value of the heat capacity ratio by using a special calculator (i.e. Calculator Based Laboratory (CBL)). A short pulse signal is produced at an open end of a tube and the echo of reflected signal is measured by a microphone and CBL/TI92 graphing calculator. The main disadvantage of proposed method is that the apparatus needs additional equipment such as function generator, data acquisition board and a good knowledge of signal processing [13].

The previously described methods and procedures used to determine the heat capacity ratio are considered somewhat complex and time consuming. The literature review shows that in spite of this, these methods are still in use in laboratories, but the problem of specific heat ratio definition is still an important issue.

Other works [14-17] illustrate several techniques, based on acoustic measurement that are applicable to measure several physical and chemical parameters such as: density of gases, temperature, displacement and concentration. These methods are based on creating a standing wave within a resonance tube (forced or free resonance mode of operation) and then the physical phenomenon based on acoustic measurement is acquired by a microphone and signal processing is performed using LabVIEW software [16].

In this work, the resonance tube method is operated in free resonance mode and used to measure the heat capacity ratio. The main advantage of the proposed method is the simplicity in the measurements and the clearness of the procedure. In addition, the measurement of the heat capacity ratio can be performed regardless of the surrounding ambient temperature as the speed of sound formula includes the temperature change.

\section{THEORETICAL BACKGROUND}

Waves of the same frequency are called coherent, if the vibrations are due to them, at each point in space, the waves have a constant phase difference.

When adding coherent waves of the same direction, the phenomenon of wave interference occurs, in which the redistribution of energy in space is increased at some points, at others points, they weaken each other.

Standing waves occur as a result of the interference of two waves propagating towards each other. Both of these waves, in contrast to standing waves, are called traveling waves.

In practice, a standing wave occurs if a well-reflecting obstacle is placed on the path of a traveling (direct) wave perpendicular to the direction of propagation. A wave (direct) falling on an obstacle and a reflected (backward) wave running towards it, overlapping each other, form a standing wave.

If a standing wave propagates in a pipe with air closed at both ends, then the length of the column is $L=n \lambda / 2$, where $n$ is an integer $(n=1,2,3, \ldots)$, it can be shown that acoustic resonance occurs. Indeed, in this case a knot is formed at one end of the pipe.

The distance between adjacent nodes is $\lambda / 2$, and since the length of the column is $n \lambda / 2$, a node is formed at the other fixed end.

If the length of the resonating column is changed by the value of $\lambda / 2$, since the length of the air column remains equal to the whole number of wavelengths, then the resulting column will also resonate.

Therefore, the smallest difference in the lengths $l$ of air columns of a gas, at which resonance occurs, is equal to:

$$
l=\frac{\lambda}{2}
$$

Hence

$$
\lambda=\frac{l}{2}
$$

With a certain frequency of the sound wave, the speed of sound $\mathrm{C}$ is given as:

$$
\mathrm{V}=\lambda f=2 f l .
$$

or the resonance frequency is given by:

$$
\mathrm{f}=\mathrm{V} / 2 \mathrm{~L} \text {. }
$$

The propagation of a sound wave in a gas (air) occurs adiabatically, since compression and rarefaction in a gas replace each other. It is known that the speed of propagation of a sound wave in gases depends on the adiabatic index. The speed of sound in gases is determined by the formula [8]:

$$
\mathrm{V}=\sqrt{\frac{\gamma \mathrm{RT}}{\mathrm{M}}}
$$

From the Eq. (5), we can find the formula to calculate the $\gamma$ as follows:

$$
\gamma=\frac{V^{2} \cdot \mathrm{M}}{R \cdot T}
$$

Substituting the value of frequency from Eq. (4) in Eq.6, then we have:

$$
\gamma=\frac{(2 f l)^{2} \cdot \mathrm{M}}{R \cdot T}
$$

To characterize the thermal properties of gas, like any other body, a special value which is called " heat capacity" is determined.

The heat capacity of the body is the amount of heat that needs to be brought to it or taken away from it to change its temperature $(\Delta \mathrm{T})$ by $1 \mathrm{~K}$ :

$$
C=\frac{\Delta Q}{\Delta T}
$$

The heat capacity per unit mass of a substance is called the specific heat capacity(ratio). It, obviously, characterizes not the body, but the substance of which this body consists. The heat capacity attributed to one mole is called the molar heat capacity.

There is an obvious relationship between the specific heat capacity and molar heat capacity: 


$$
\gamma=\frac{C}{M}
$$

The process is called adiabatic if in this process there is no heat exchange between the gas and the environment (the walls of the vessel are insulated). If the adiabatic process is in equilibrium (at each moment of time, pressure (P) and temperature (T) are the same throughout the volume), then it is described by the Poisson equation.

$$
P V^{\prime}=\text { const } \text {. }
$$

According to the first law of thermodynamics

$$
\Delta Q=\Delta U+\Delta A
$$

Consider one mole of gas, if $\mathrm{V}=$ const, then $\Delta \mathrm{A}=0$ and, therefore, $\mathrm{CV}=\Delta \mathrm{U} / \Delta \mathrm{T}$. If $\mathrm{P}=$ const, then $\Delta \mathrm{A}=\mathrm{P} \Delta \mathrm{V}=\mathrm{R} \Delta \mathrm{T}$, since the equation of state $(\mathrm{PV}=\mathrm{RT})$ is valid in the initial and final state of the gas.

Consequently,

$$
C_{p}=\frac{\Delta U}{\Delta T}+R \frac{\Delta T}{\Delta T}=C_{V}+R
$$

Thus, the molar heat capacities $\mathrm{Cp}$ and $\mathrm{Cv}$ for an ideal gas are related by the relation:

$$
C_{p}=C_{v}+\mathrm{R}
$$

Since, $\mathrm{Cp}>\mathrm{Cv}$, since at $\mathrm{V}=$ const, all the heat transferred to the gas goes only to change the internal energy of the gas, while at $\mathrm{P}=$ const the heating of the gas is inevitably accompanied by its expansion. At the same time, the gas does work, for which it is necessary to inform it of an additional amount of heat.

In the molecular-kinetic theory of gases, it is shown that, $C_{v}=\frac{i}{2} R$ where $\mathrm{i}$ is the number of degrees of freedom of a single molecule, the number of independent coordinates by which the position and orientation of the molecule in space is uniquely determined.

For monatomic molecules, $\mathrm{i}=3$ (for example, inert gases), for two atomic rigid molecules (the distance between molecules is constant) $i=5$.

Such are the $\mathrm{N}_{2}$ and $\mathrm{O}_{2}$ molecules in the air at room temperatures and, therefore, for them:

$$
\gamma=\frac{C_{p}}{C_{v}}=\frac{C_{v}+R}{C_{v}}=1+\frac{R}{C_{v}}=\frac{i+2}{i}=1,4
$$

Thus, to determine the adiabatic index or specific heat ratio, it is sufficient to measure the temperature of the gas and the speed of sound propagation within a resonance tube which are a function of of the frequency of created standing wave within a tube (the molar mass is assumed to be known).

\section{METHODOLOGY}

Measuring the speed of sound in gas $\mathbf{C}$ at a certain temperature $\mathrm{T}$ and knowing the molar mass of gas $\mathrm{m}$, one can calculate one of the most important thermodynamic characteristics, i.e. the adiabatic index $\gamma$.

In this work, the speed of sound is measured by the method of standing waves generated in a column of gas enclosed in a pipe. In one end of the tube a microphone is fixed, where a speaker is mounted in the other end of the tube. When a free resonance mode is applied, a standing wave in is created within resonance tube, the oscillation frequency coincides with one of the natural frequencies of the air column (the phenomenon of resonance). The U-shape resonance tube is used in work [8], the authors utilized U-shape tube to build an acoustic temperature transducer based on resonance mode of standing wave generation within a tube filled by a medium.

On the other hand, the U-shape tube is utilized in work [17] to measure the density of liquids.

In this work, the authors will utilize the same shape to determine the ratio $\mathrm{Cp} / \mathrm{Cv}$ using free mode of acoustic resonance detector. The schematic design of the resonance tube is shown in Figure 1.

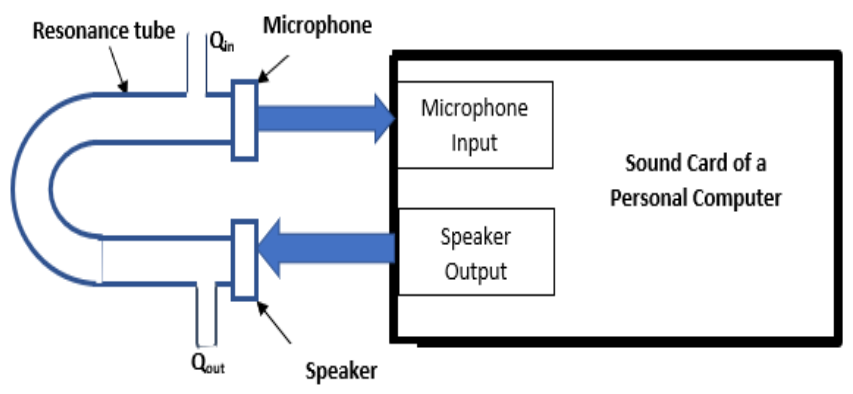

Figure 1. The schematic design of acoustic resonance detector for determination of the ratio $C_{p} / C_{v}$

The operation principle of the detector is as follows. The tube is filled by a sample of gas under test and this can be done by introducing a flow of gas via capillary tubes with a constant flow rate $(0.5 \mathrm{~L} / /$ hour $)$. Then it is necessary to adjust the sound card of the computer system so the microphone "listens" to the speaker. In this case a sound with a fixed frequency will be heard because of the effect of resonance.

The signal of microphone can be introduced for further processing using any software which is capable to measure the sound frequency.

LabVIEW is utilized to make measurement of input parameters required to determine the ratio $\mathrm{C}_{\mathrm{p}} / \mathrm{C}_{\mathrm{v}}$.

LabVIEW is a program designed to create an experiment using a computer. It is used by many scientists and engineers around the world. LabVIEW provides the ability to work with numeric, binary, string data, as well as groups and arrays. In addition, it is possible to output the result in the form of a graph, that is, the construction of graphs of various functions [18].

The graphical programming language used by LabVIEW is based on a data stream architecture. The sequence of execution of operators in such languages is determined not by the order of their sequence (as in imperative programming languages), but by the availability of data on the inputs of these operators.

Using the LabVIEW open programming environment to implement the developed algorithms and exchange data with modeling tools, it is possible to modernize development tools and reduce time costs at all stages of the product life cycle [19].

The block diagram of the designed software is shown in Figure 2.

The experimental procedure is described by the following steps:

1. It is recommended to configure the sound card input and output parameters, so the echo signal is produced.

2. The signal from the microphone is acquired by 
LabView VI which is called MIC Read VI.

3. The output of MIC Read VI is then connected to Tone Measurement VI.

4. The Tone Measurement VI measures the frequency of created standing wave.

5. The measured frequency is applied in Eq. (6) and the specific heat ratio is calculated using mathematical VIs available by LabVIEW.

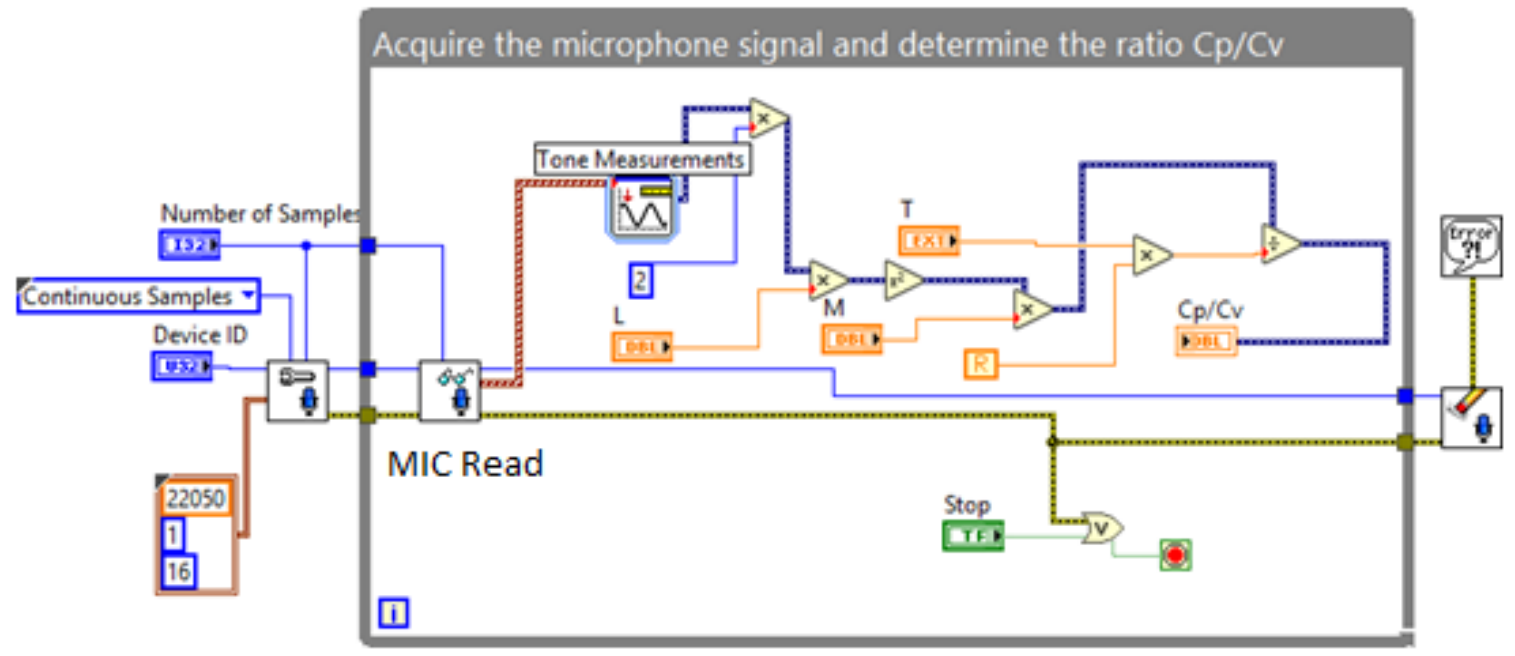

Figure 2. The VI design to acquire the microphone signal and determine the ratio $C_{p} / C_{v}$

\section{EXPERIMENTAL RESULTS}

The experimental results are obtained by calculating the specific heat ratio for different gases at different temperatures and by variation the different length of the resonance tube (L) as shown in Table 1 .

Table 1. Experiment conditions

\begin{tabular}{|c|c|c|}
\hline Gas & $\mathrm{t},{ }^{\circ} \mathrm{C}$ & $\mathrm{L}, \mathrm{mm}$ \\
\hline Air, $\mathrm{CO}_{2}, \mathrm{He}$ & $20,25,30$ & $50,100,150,200,250,350$ \\
\hline
\end{tabular}

This allows to make measurement of frequency of the created standing wave in different conditions. Since the frequency of the created standing wave depends at the media filled within the tube, the ambient temperature and the length of resonance tube which defines the distance between the acoustic transmitter and acoustic receiver.

The experimental results of the average values of specific heat ratio $\gamma$ for different gases and at different temperature points are presented in Table 2.

Table 2. The values of specific heat ratio $\gamma$

\begin{tabular}{|c|c|c|c|}
\hline \multirow{2}{*}{$\begin{array}{c}\text { Sample } \\
\text { under test }\end{array}$} & \multicolumn{3}{|c|}{ The average $\boldsymbol{\gamma}$ at different temperature } \\
\cline { 2 - 4 } & $\boldsymbol{\gamma}_{\mathbf{2 0}}$ & $\boldsymbol{\gamma}_{\mathbf{2 5}}$ & $\boldsymbol{\gamma}_{\mathbf{3 0}}$ \\
\hline Air & 1.39 & 1.39 & 1.4 \\
\hline $\mathrm{CO}_{2}$ & 1.28 & 1.27 & 1.28 \\
\hline $\mathrm{He}$ & 1.65 & 1.65 & 1.66 \\
\hline
\end{tabular}

Figure 3 shows the plot between the length of resonance tube and the frequency of created standing wave within a tube for different gases and at different temperatures.

Measuring the frequency of created standing wave allows to calculate the speed of sound at different temperatures and for various types of gases filled the resonance tube. The experimental results that describe the variation of sound speed for the three gases (at different temperature points and when the length of the tube varies from $50 \mathrm{~mm}$ to $350 \mathrm{~mm}$ ) is shown in Figure 4
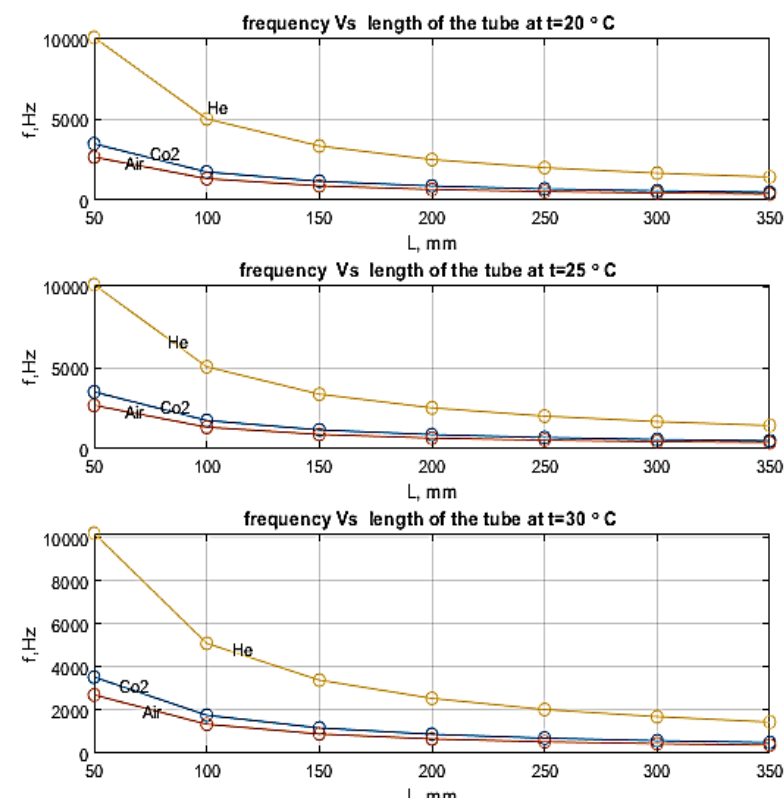

Figure 3. The plot(s) between the frequency of standing wave and the length of the tube at different temperature points

The experimental results show that the length of the tube has a significant effect on the frequency measurement (which is limited by the features of the sound card). So, to overcome this effect it is recommended to select the length of the resonance tube between $200 \mathrm{~mm}$ and $250 \mathrm{~mm}$.

Concerning the relative error by using the experimental results obtained for the samples of gases under test, the maximum relative error is about $2 \%$. It is important to note that environmental effects such as temperature, humidity or vibration may have a minimum negligible effect on the detector performance. That is because the resonance tube may 
be made by of any isolating material with any wall thickness.
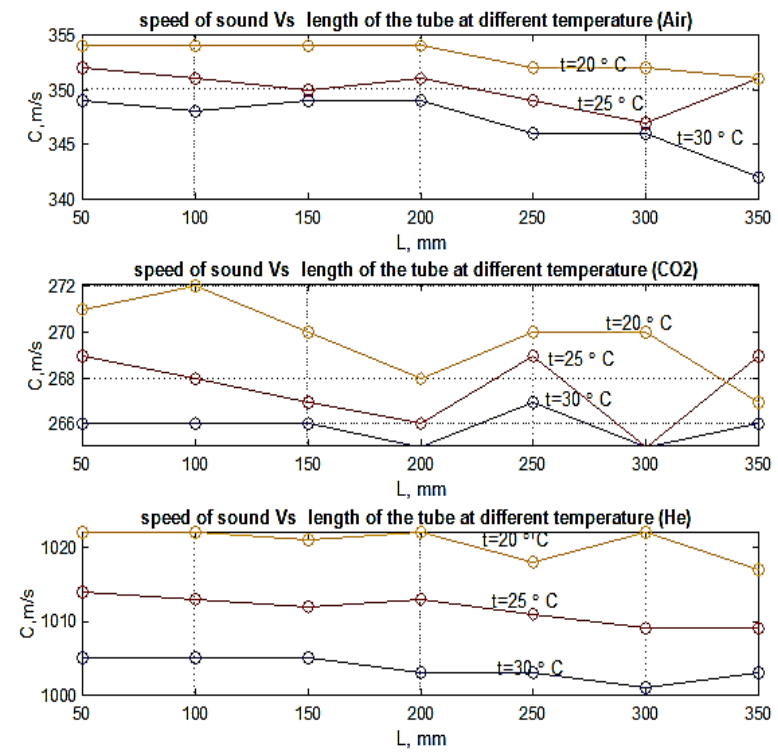

Figure 4. The variation of speed of sound vs. the length of the tube (at different temperature points)

\section{CONCLUSION}

This paper presents a simple computerized technique for measuring the specific heat ratio based on acoustic resonance measurement. Experimental results show that the specific heat ratio of gases (air, carbon dioxide, helium) can be measured and calculated using a simple resonance tube filled by the sample gas undertest.

\section{REFERENCES}

[1] Ceviz, M.A., Kaymaz, I. (2005). Temperature and airfuel ratio dependent specific heat ratio functions for lean burned and unburned mixture. Energy Conversion and Management, 46(15-16): 2387-2404. https://doi.org/10.1016/j.enconman.2004.12.009

[2] Huang, B.J., Chang, J.M., Wang, C.P., Petrenko, V.A. (1999). A 1-D analysis of ejector performance. International Journal of Refrigeration, 22(5): 354-364. https://doi.org/10.1016/S0140-7007(99)00004-3

[3] Lau, J.H. (ed.). (1997). Canadian Nuclear Society, Toronto, Ontario (Canada). pp. 321-341.

[4] Gauthier, T., Weidner, S., Martinez, B. (2017). Pressure measurements using pressure sensitive paint in supersonic flow. Instrumentation Mesure Métrologie, 16(1-4): 115-141. https://doi.org/10.3166/i2m.16.14.115-141

[5] Krause, D.E., Keeley, W.J. (2004). Determining the heat capacity ratio of air from "Almost Adiabatic" compressions. The Physics Teacher, 42(8). https://doi.org/10.1119/1.1814323

[6] Alsabbah, S., T. Mughrabi, (2008). Neural networkbased waveguide acoustic gas detector. 2008 5th International Symposium on Mechatronics and Its Applications, IEEE https://doi.org/10.1109/ISMA.2008.4648867
[7] Gupta, A.K., Kumar, M., Panda, D., Sahoo, R.K. (2018). Experimental analysis to predict the performance of a plate fin heat exchanger at cryogenics temperature. Instrumentation Mesure Métrologie, 17(2): 315-329. https://doi.org/10.3166/I2M.17.315-329

[8] Younes, T.M., Alia, M.A.K., Al-Sabbah, S. (2010). Acoustic temperature transducer. Sensors \& Transducers Journal, 119(8): 46-57.

[9] Rebillot, P.F. (1998). Determining the Ratio Cp/CV using Rucchart's Method. Physics Department, The College of Wooster, Wooster, Ohio 44691 April 30, 1998.

[10] Lucas, S. (2010). Measurement of $\mathrm{Cp} / \mathrm{Cv}$ for argon, nitrogen, carbon dioxide and an argon + nitrogen mixture. University College London.

[11] Qin, J.L., Shang, S.P. (2019). Laboratory acoustic measurement of prorocentrum donghaiense concentrations. Instrumentation Mesure Métrologie, 18(2): 181-188. https://doi.org/10.18280/i2m.180205

[12] Branca, M., Soletta, I. (2007). Cp /Cv ratios measured by the sound velocity method using calculator-based laboratory technology. J. Chem. Educ., 84(3): 462. https://doi.org/10.1021/ed084p462

[13] Halliday, D., Resnick, R., Walker, J. (2008). Fundamentals of Physics. - 8th ed. Hoboken (N. J.), John Wiley \& Sons, Inc.

[14] Younes, T.M. (2012). Computer-based acoustic detector for determining the type and concentration of a solution. Journal of Applied Research and Technology, 10: 859864.

[15] Alia, M.A.K., Younes, T.M., Zalata, M.A. (2011). Development of equivalent virtual instruments to PLC functions and networks. Journal of Software Engineering and Applications, 4: 172-180. https://doi.org/10.4236/jsea.2011.43019

[16] Younes, T.M. (2019). Novel approach of non-linearity analyses of resistive temperature sensors. Revue d'Intelligence Artificielle, 33(2): 159-164. https://doi.org/10.18280/ria.330212

[17] Younes, T.M., AlKhedher, M.A., Shgier, K.A., Al Taweel, F. (2019). U-shape acoustic liquid densitometer. Instrumentation Mesure Metrologie, 18(2): 123-128. https://doi.org/10.18280/i2m.180205

[18] Younes, T.M. (2019). Novel approach of non-linearity analyses of resistive temperature sensors. Revue d'Intelligence Artificielle, 33(2): 159-164. https://doi.org/10.18280/ria.330212

[19] Ferdinand P., Pouillé F., Ricque B., Bourbotte J.M., Hamrita H., Kondrasovs V., Makil H., Maurin L., Rougeault S., Cheymol G., Damian F., Duval D., Jaboulay J.C., Le Tutour P., Maskrot H., Barbot L., Haquet J.F., Journeau C., Souquet Q., Villard J.F., Musoyan G., Brovchenko M., Duhamel I., Fourrez S., Helleux G., Pichon L., Ouerdane Y. (2017). When the remote measurement instrumentation works to improve the monitoring of the reactor building of nuclear power plants in case of severe accident. Instrumentation Mesure Métrologie, 16(1-4): 183-211. https://doi.org/10.3166/i2m.16.1-4.183-211

\section{NOMENCLATURE}

n An integer number

$\mathbf{C}_{\mathbf{v}} \quad$ Heat capacity at constant volume, J/K 
$\mathbf{C}_{\mathbf{p}} \quad$ Heat capacity at constant pressure, $\mathrm{J} / \mathrm{K}$

C Molar heat capacity, J/mol

V Speed of sound, $\mathrm{m} / \mathrm{s}$

L Length of the tube, $m$

f Frequency of standing wave, $\mathrm{s}^{-1}$

R Ideal gas constant

T Atmospheric temperature, $\mathrm{k}$

$\Delta \mathbf{T} \quad$ Change in temperature of the body, in kelvin

$\boldsymbol{l}$ The smallest difference in the lengths of air columns of a gas, in mm

M Molecular weight of gas
$\Delta \mathbf{Q}$ The amount of heat imparted to the gas

$\Delta \mathbf{U} \quad$ The change in the internal energy of the gas, $\mathbf{J}$

$\Delta \mathbf{A} \quad$ The work done by the gas on the external bodies. $\mathrm{J} / \mathrm{s}$

P Pressure, $\mathrm{Pa}$

\section{Greek symbols}

$\lambda \quad$ The wave length of the standing wave

$\gamma \quad$ Specific heat ratio 